\title{
Transmission block in terminal nerve twigs : a single fibre electromyographic finding in man
}

\author{
ERIK STÅLBERG AND BARBARA THIELE \\ From the Department of Clinical Neurophysiology, \\ Academic Hospital, Uppsala, Sweden
}

SUMMARY Single fibre electromyography has been performed in patients with partial nerve $\frac{\frac{5}{\omega^{5}}}{\frac{5}{6}}$ lesions, amyotrophic lateral sclerosis, progressive spinal muscle atrophy, muscular dystrophy, and distal hereditary myopathy. The recorded action potentials were often more complex than in the normal muscle due to increased fibre density in the motor unit and the individual spike components showed a large jitter and occasional blockings. Sometimes two or more spikes in a complex disappeared and reappeared simultaneously upon successive discharges. This phenomenon, called 'paired blocking', has been further investigated. The jitter of the blocking potentials in relation to the rest of the complex was large, up to $500 \mu \mathrm{sec}$. The degree of blocking increased with increasingo innervation frequency until it eventually proceeded to total block during continuous activity. Some- $-\omega_{\pi}^{-}$ times a slight effect on blocking was seen after edrophonium. This type of block is probably localized in the terminal nerve twigs, perhaps in newly formed sprouts. The phenomenon of neurogeq on blocking may contribute to the fatigue clinically experienced in different denervation-reinnervati $\bar{B}$ in cases.

With a multielectrode technique described by Ekstedt and Stålberg (1963), Ekstedt (1964), and Stålberg (1966), the electrical activity was recorded from voluntarily activated human single muscle fibres. Normally the muscle fibres, belonging to one motor unit, are separated from each other so that an electrode of the type used in this investigation, $25 \mu$ in diameter, records activity from only one muscle fibre in a certain motor unit in about $70 \%$ of the insertions. The uptake radius of the electrode is about $200 \mu$. In about $30 \%$ of the insertions two or, exceptionally, more muscle fibres belonging to the same motor unit can be recorded. The action potentials from different fibres are in these cases always coupled to each other with a small variability in the interpotential interval from discharge to discharge-the jitter (Ekstedt, 1964; Stålberg, Ekstedt, and Broman, 1971). In the normal muscle there is never any blocking of one or the other potential in such a potential pair. However, in myasthenia gravis, for example, the jitter is increased and occasional blockings of one or the other of the potentials are seen, more frequent in patients with pronounced symptoms (Ekstedt and Stålberg, 1967; Blom and Ringqvist,
1971). If activity from three fibres from motor unit happens to be recorded in a mysthenic muscle, it can be seen that the blocking appears independently in the different fibres (Fig. 1a).

In different pathological conditions, especiallye in lower motor neurone disorders, there is ang increased fibre density in the motor unit due to peripheral sprouting (Wohlfart, 1957; Kugelberg Edström, and Abbruzzese, 1970) and therefore the activity from many muscle fibres belonging to one motor unit can be recorded from the same electrode. The number of simultaneouslys recorded fibres in the same motor unit is typically between three and 10 . In such a complex the jitter between individual muscle fibre potentials is increased and blockings are seen.

During the study of these cases with single fibre electromyography it was sometimes seen? that in the compound action potentials two of more blocking spike components were intimately. coupled and none of these spikes blocked indes pendently of the other(s). From now on this wils be called 'paired blocking' (Fig. 1B). Thiß investigation is aimed at studying the nature of this kind of blocking. 


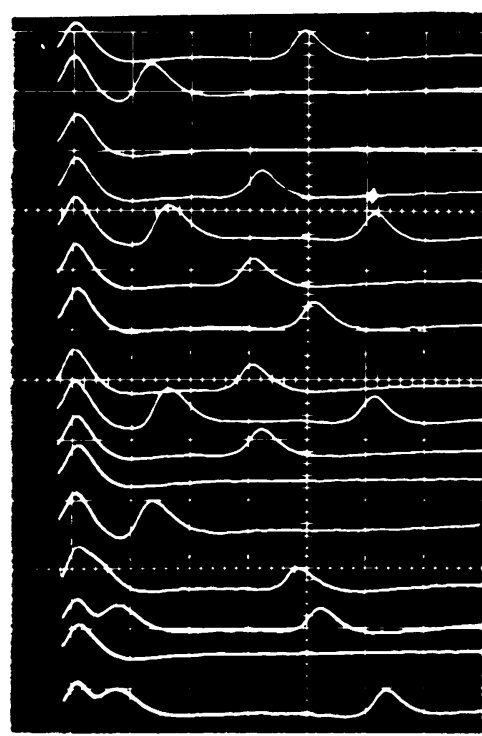

A

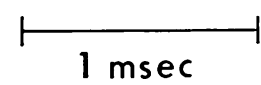

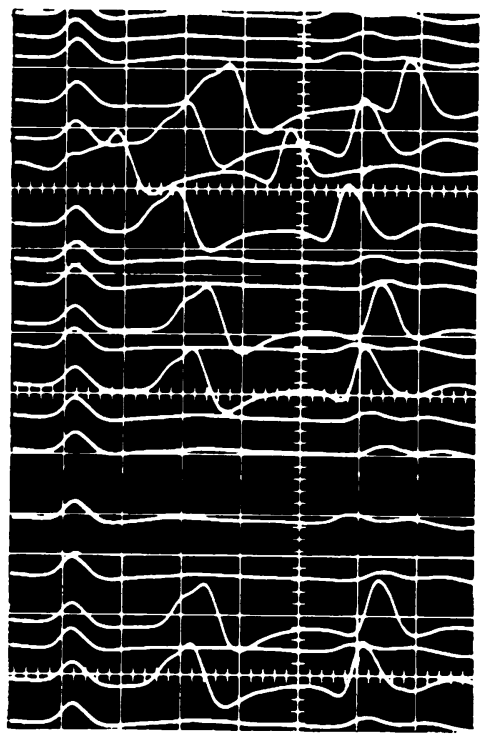

B
FIG. 1. A. Recording from three muscle fibres belonging to the same motor unit in myasthenia gravis. The position of the sweep is continuously altered downwards. The second and third action potential are independently blocking at random. Jitter increased in both. B. Recording from four muscle fibres in muscular dystrophy. The second component (generated by two muscle fibres) and the third are frequently blocking but always simultaneously, 'paired blocking'.
The phenomenon of 'paired blocking' was especially looked for in the following subjects: three patients with partial ulnar nerve lesion (compression in sulcus ulnaris), five patients with amyotrophic lateral sclerosis, three patients with progressive spinal muscular atrophy, two patients with progressive muscular dystrophy, and two patients with distal hereditary myopathy. Furthermore, three healthy men who had earlier served repeatedly as experimental subjects were studied in the $\mathrm{m}$. extensor digitorum communis (III) at the place where hundreds of earlier needle insertions had been made. The picture had changed in this muscle from normal to a pattern indicating reinnervation. It seems likely that the traumata caused by the needle had caused a certain degree of peripheral nerve lesion accompanied by reinnervation.

Usually the $\mathrm{m}$. extensor digitorum communis was studied except for the patients with ulnar nerve lesions where the $\mathrm{m}$. abductor digiti quinti was investigated. Sometimes also $\mathrm{m}$. tibialis anterior, $\mathrm{m}$. quadriceps, and $\mathrm{m}$. biceps were studied.

Recording was made with a multielectrode with two electrode surfaces ( $25 \mu$ in diameter) in a hole in the side of the needle near the tip. A cutaneous pad electrode served as indifferent reference. The potentials were displayed on a dual beam oscilloscope (Tektronix 565) and on a storage oscilloscope (Tektronix 549) and fed into an analogue tape recorder (Akai X330). The analysis was always made on the storage oscilloscope, sometimes on running film. Sometimes the action potentials were recorded on an ink jet recorder (Mingograf type 30B). For measurements of the jitter, a time interval counter (Hewlet Packard 5245 L with time interval unit 5262 A) was used, feeding data in digital form to a magnetic tape recorder (Dymec 2546) which served as input medium for a computer (CDC 3600).

\section{RESULTS}

The phenomenon of 'paired blocking' was observed in all the above-mentioned cases, especially in the early phase of reinnervation or in the progressive state of a disease, but in relation to recordings with single fibre blockings is was relatively rare. When the degree of blocking was low, there was no doubt that the blocking potentials belonged to the same motor unit as the other potentials in the complex under study. In cases where there were frequent blockings, however, difficulties could arise in determining whether the potentials belonged to another motor unit and only incidentally appeared simultaneously with the action potentials under study. However, by using very slight contraction so that the compound action potential was the only activity to be recorded, it could be settled that the partially blocking potentials, when they appeared, did this together with the compound potentials and never in isolation (Fig. 2). 


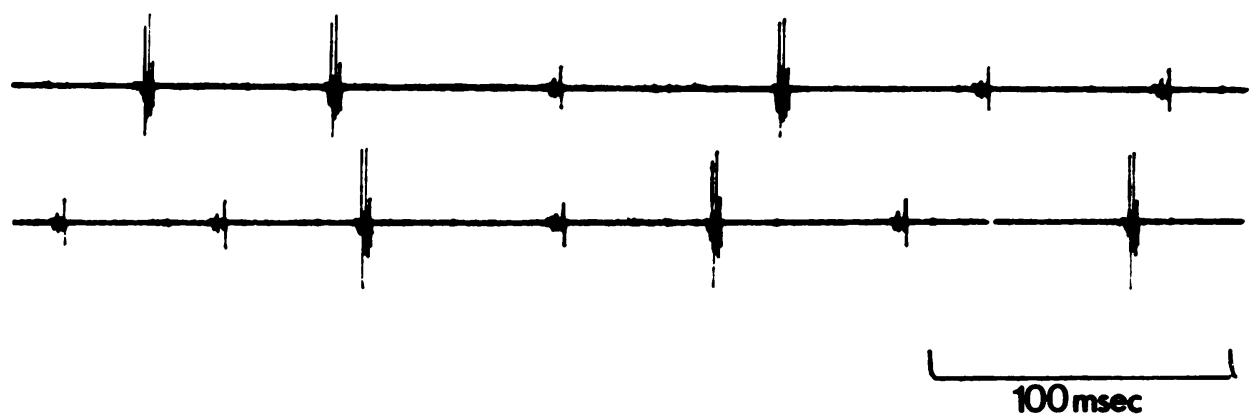

FIG. 2. Continuous recording of a compound action potential where two spikes (the largest) block together. The two blocking potentials never appear without the rest of the action potential complex. Potentials slightly retouched.

Often the blocking potentials were late, but sometimes they belonged to the middle or early part of the compound action potentials (cf. Fig. 6). The jitter between the blocking potentials was within the normal range or increased but was never smaller than normal. However, the blocking potentials jittered as a unit in relation to the other spikes in the complex with a jitter that regularly was greater than the internal jitter between the blocking potentials and could measure $500 \mu \mathrm{sec}$ (expressed as MCD, Stålberg et al., 1971). There was no correlation between this jitter and mean interspike interval. Sometimes the intervals between the blocking and nonblocking part of the complex showed a systematic change for the first three to five consecutive discharges in periods without blockings, usually first a shortening then sometimes an increase again before blocking (Fig. 3). The amount of this change was not correlated with the mean interspike interval.

EFFECT OF EDROPHONIUM The degree of blocking was studied in 10 experiments before and after intravenous injection of edrophonium (Tensilon), first $2 \mathrm{mg}$ and after 45 seconds another 2 or $8 \mathrm{mg}$. In three experiments the jitter and degree of blocking were unchanged. In seven experiments there was a decreased blocking frequency and jitter, but the effect was in most cases less than what is usually seen in myasthenia gravis where the blockings can often be totally suppressed. In Fig. 4 is shown the recording where the most pronounced edrophonium effect was obtained.

EFFECT OF DIFFERENT INNERVATION FREQUENCIES In four experiments the innervation frequency was kept constant at about 10 impulses/second

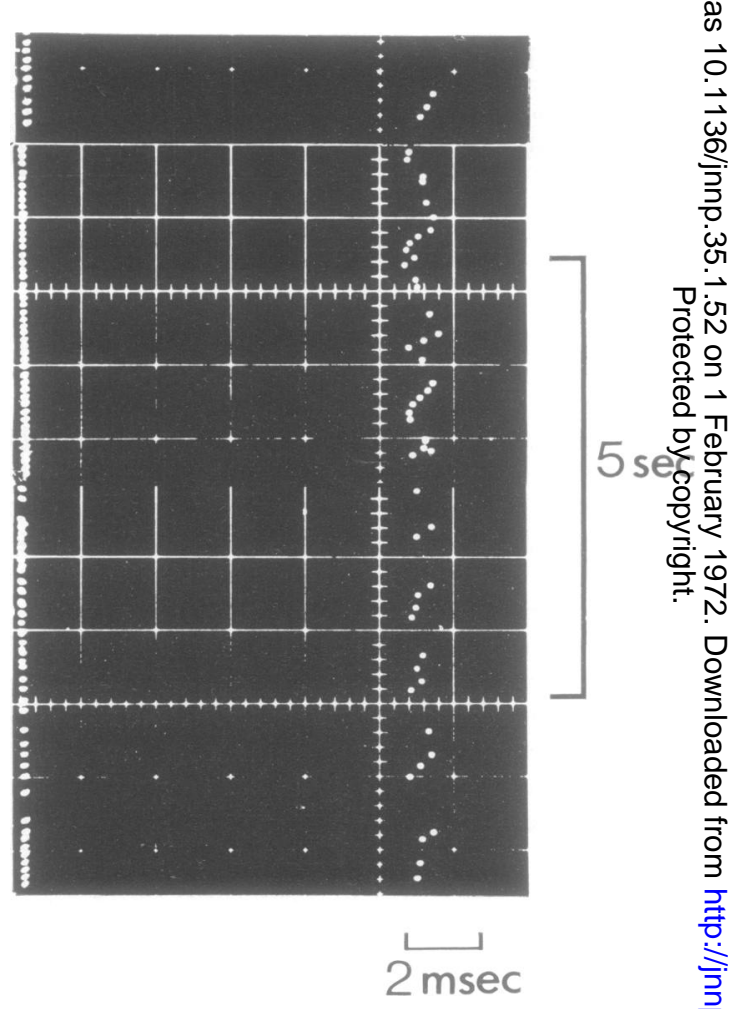

FIG. 3. Jitter in neurogenic block. The non-blocking part of the action potential triggers the sweep and is represented by the left dot, the blocking part by the right dot. The position of the sweep is continuouslys altered downwards. At blocking, the right dot is missing. The distance between the non-blocking anct blocking part is about $11 \mathrm{msec}$ and shows a tendenc to systematic changes during periods of consecutive discharges without blocking.

for 10 minutes. In all these cases there was 5 tendency to increased degree of blocking duringo this time, in one case from $20 \%$ to total block after 1.5 minutes (Fig. 5A). 

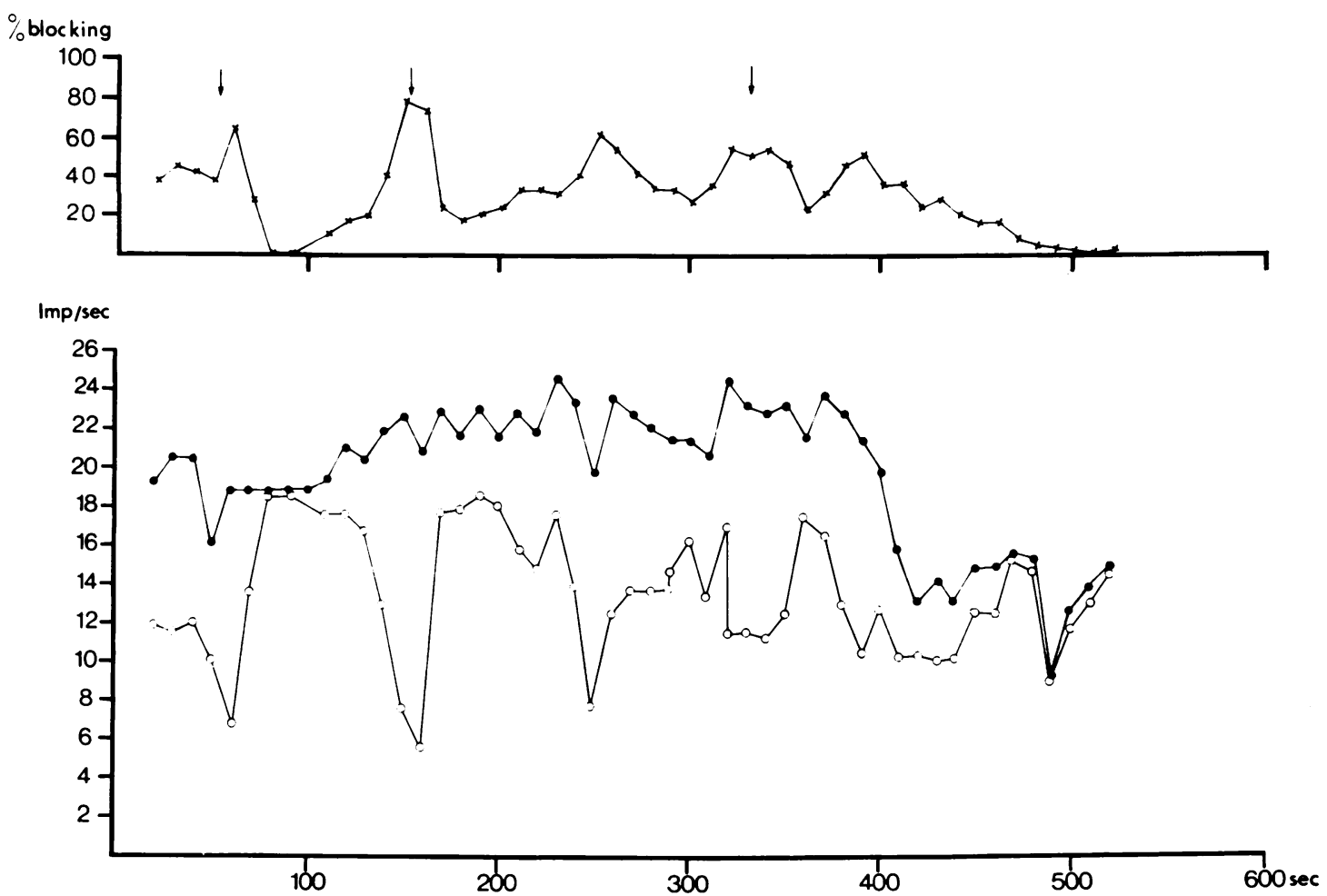

FIG. 4. Effect of edrophonium. The recording was made in $m$. extensor digitorum III in a patient with amyotrophic lateral sclerosis. Open circles indicate mean discharge rate during 10 seconds for blocking spikes and filled circles corresponding value for non-blocking spikes in the compound action potential. Upper part (crosses) shows degree of blocking in per cent. Injection of $2 \mathrm{mg}$ edrophonium (each arrow) is followed by a reduction in the degree of blocking (somewhat more than usually seen). When the innervation frequency is reduced at the end of the experiment (after 400 seconds) from about 22 to about 15 impulses/sec the degree of blocking decreases towards zero.

In eight experiments the innervation frequency was kept constant for a few minutes at different frequency levels or slowly changed between 4 and 15 impulses/second. With a delay of about 20 seconds the blocking increased at higher frequency and decreased at lower (Fig. 5B). The change in degree of blocking was somewhat different for different motor units. The jitter increased slightly during continuous activity.

\section{DISCUSSION}

In the normal muscle there is a high safety factor in the neural and neuromuscular transmission where thousands of impulses can be transmitted during continuous activity without signs of decreased accuracy (Stålberg et al., 1971). In early periods of progressive disorders with sprouting, compound action potentials are recorded (in the ordinary EMG as 'giant potentials' or 'reinnervation potentials') with an increased jitter and blockings. In this study inter- est was focused on the phenomenon of 'paired blocking'. The phenomenon is supposed to take place in the muscle and not be caused by such mechanisms as occasional synchronized activity between two anterior horn cells, as it is seen also in disorders with only peripheral engagements. Neither could it be explained as an occasionally appearing double discharge, as the blocking and non-blocking components were quite different in shape. Furthermore, the blocking part sometimes appears less than $1 \mathrm{msec}$ from the rest of the potential-that is, within the refractory period of nerve and muscle fibre and therefore an ectopic focus in the terminal nerve tree can also be excluded. Are the blocking potentials generated by hyperexcitable muscle fibres, mechanically triggered by adjacent muscle fibres? This seems unlikely, because when the electrical activity from the non-blocking fibres is recorded, usually less than 5 msec have elapsed since the depolarization in the end-plate region. (The recording is usually made less than $20 \mathrm{~mm}$ from the end-plate 

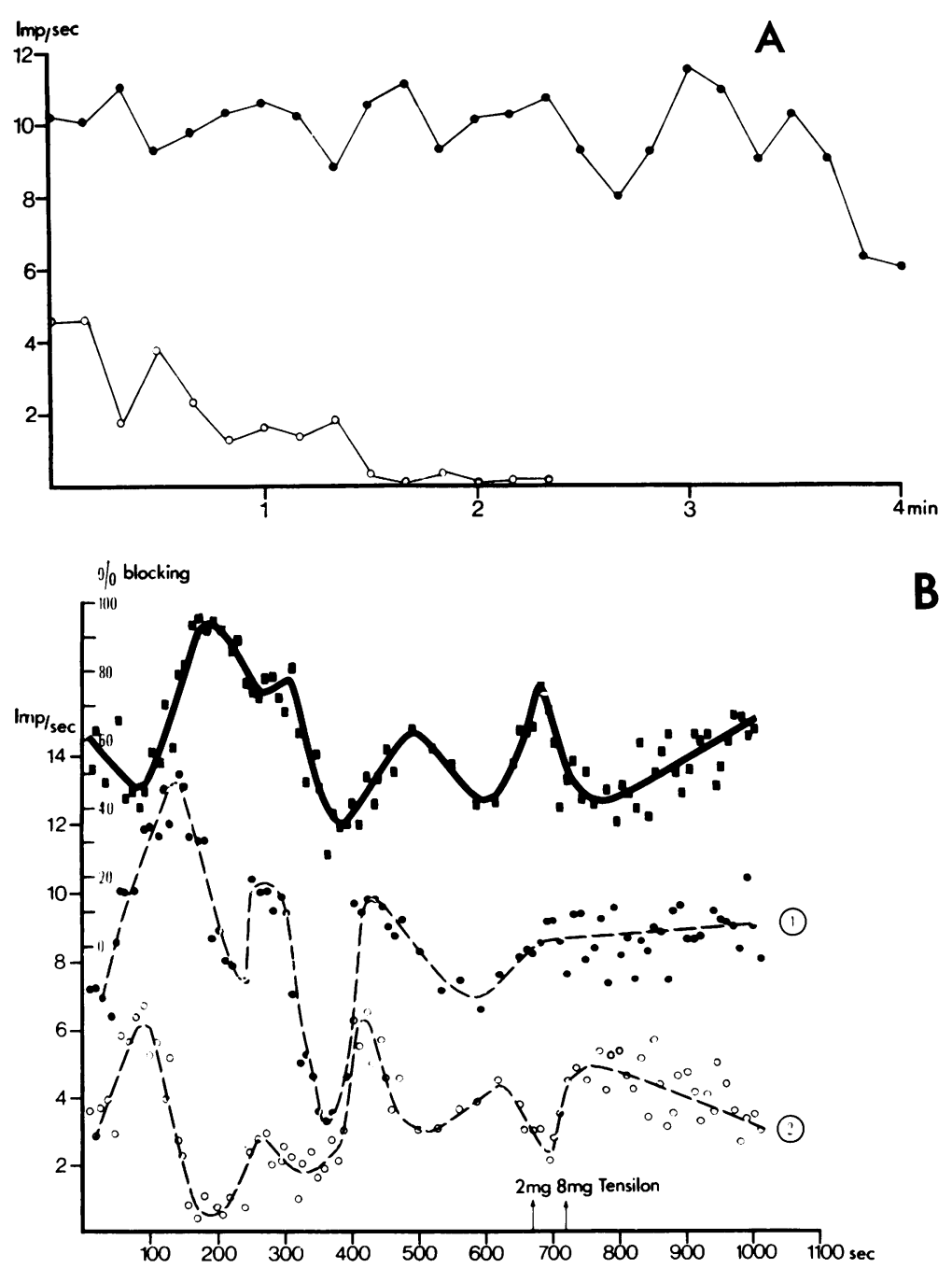

FIG. 5. Effect of activity. A. Record from a patient with ALS. Filled circles indicate mean frequency during 10 seconds for the non-blocking part and open circles for blocking part of a compound action potential during continuous activity. The frequency of the blocking part falls to nearly zero after about 1.5 minutes due to blocking. B. Recording from a case with posttraumatic reinnervation. Filled circles (curve 1) indicate mean frequency during 10 seconds for nonblocking and open circles (curve 2) for blocking spikes in the action potential complex. Squares give corresponding degree of blocking in per cent. When frequency is slowly changed between 4 and 13 impulses/second the degree of blocking changes with a delay of about 20 seconds. A higher innervation frequency is followed by $a$ higher degree of blocking and vice versa. Effect of edrophonium 2 and $8 \mathrm{mg}$ respec- $\frac{\overline{0}}{7} \overrightarrow{\mathrm{c}}$ tively at arrows is uncertain. During the last 350 seconds the innervation frequency in curve 1 is rather constant but the degree of blocking is increasing. zone and, with the propagation velocity of about $4 \mathrm{~m} / \mathrm{sec}$ (Stålberg, 1966), the time lag will be less than $5 \mathrm{msec}$ ). At this moment the mechanical activity is just initiated and has its maximum after 40 to $80 \mathrm{msec}$. Furthermore, the extreme coupling in all blocking spikes makes mechanical factors also appear too coarse and inconsistent. Finally, at the pronounced mechanical sensitivity necessary for this explanation, the blocking fibres ought to be capable of being triggered also by percussion and other mechanical stimuli, something which was never experienced.

Thus the blocking seems to indicate a failure somewhere in the transmission of nerve impulses to the muscle fibre. Obviously it cannot be localized in the muscle fibre or the motor endplate because two or more muscle fibres are consequently blocking together. In one exceptional situation a block in one nerve fibre, one motor end-plate, or one muscle fibre could give the finding described-namely, if the recording were made from the two branches of a split muscle fibre (Ekstedt and Stålberg, 1969). However, in this situation the jitter between the blocking potentials should be very small, less than $5 \mu \mathrm{sec}$, which was not the case in these recordings. Furthermore, action potentials from many muscle fibres sometimes blocked at the same time and a multiple branching of a muscle fibre is not known. Thus the most probable explanation seems to be a neurogenic trans- $\tilde{N}$ mission failure proximal to the last branching of ${ }_{\omega}^{N}$ the nerves to the blocking fibres (B in Fig. 6) but distal to the branching point where nerve 


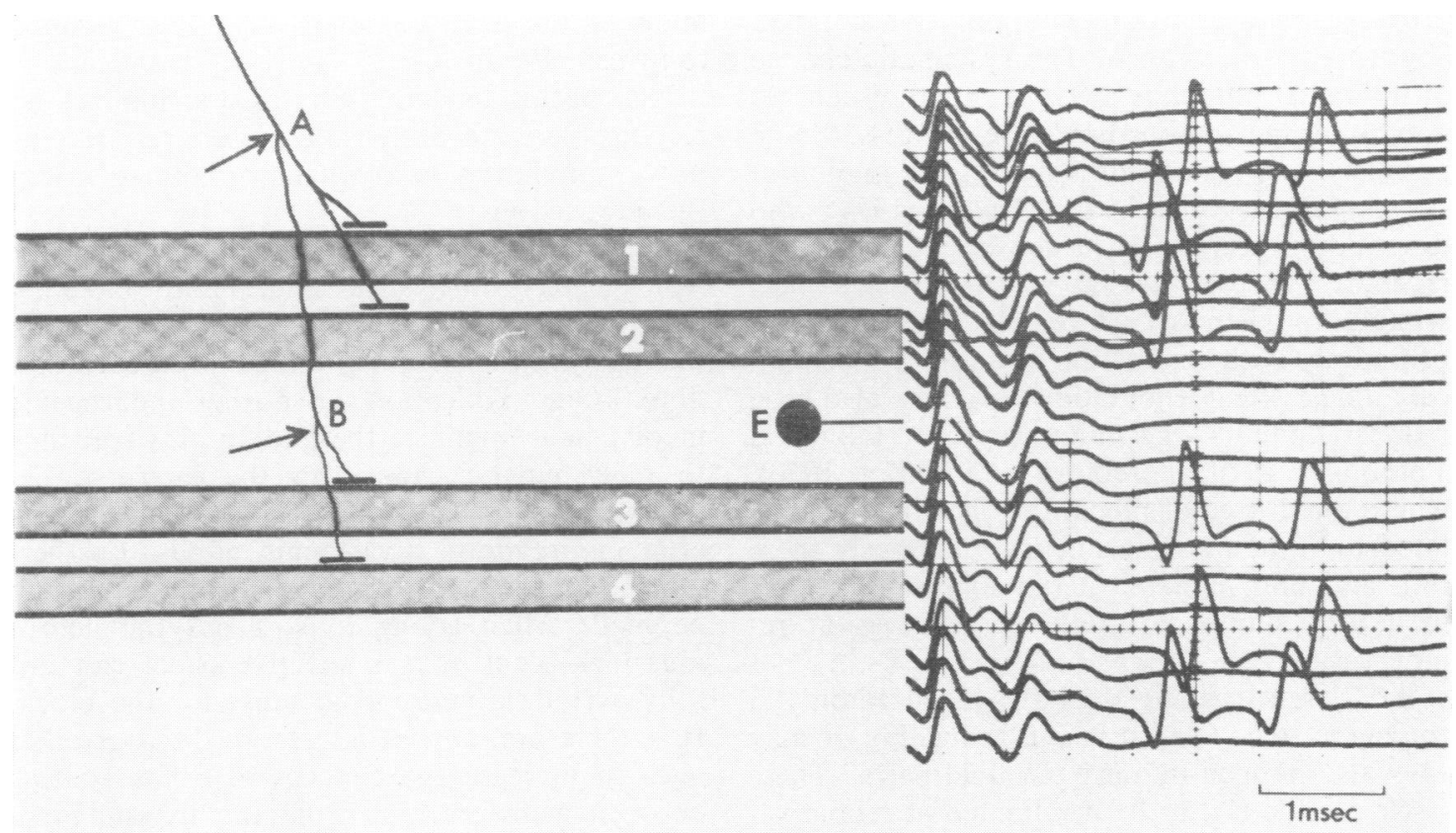

FIG. 6. Schematic drawing of recording from four muscle fibres innervated by the same nerve axon-two of them (three and four) by a sprout branching off in A. To the right an original recording from the electrode $\mathrm{E}$ of mainly four potentials, two of them blocking together (three and four). They behave as a unit which also has a large jitter in relation to the rest of the action potential complex.

branches to other muscle fibres recorded in the complex are involved (A in Fig. 6). It seems reasonable to assume that the block occurs in some branching point along this stretch. The fact that the phenomenon is seen mainly in reinnervation cases may indicate that it is associated with the newly formed nerve sprouts. These are initially not myelinated (van Harreveld, 1952; Wohlfart, 1958) and therefore transmit the nerve impulses less well than the normal twig (Gilliatt, 1966). An indication that the blocking appears in generally poorly conducting fibres is given by the fact that the blocking potentials often appear late in the compound action potential - that is, delayed in relation to the other part of the complex. The great jitter measured between the blocking potentials and the remaining part of the compound potentials also indicates an uncertainty in the nerve transmission with a variability in the nerve conduction time from discharge to discharge, a neurogenic jitter. The systematic change in the first three to five consecutive interpotential intervals directly after a period of block probably depends on continuous change in impulse propagation velocity in the nerve twig. The phenomenon is similar in nature to that described by Stålberg (1966) in muscle fibres.

The slight but clear effect of edrophonium is somewhat difficult to explain. Edrophonium is considered to be a pure cholinesterase inhibitor and should therefore have no influence upon the impulse conduction along the nerve twig. However, Okamoto and Riker (1969) have shown that edrophonium has an effect on the normal unmyelinated nerve terminals, producing especially repetitive firing and twitch potentiation. They considered this to be a direct drug action on the nerve terminal membrane and not indirectly via cholinesterase inhibition.

The finding of fatiguability and sensitivity to high frequency stimulation, similar to the findings in myasthenia gravis, remains also to be explained. Accommodation, some kind of electrolytic exhaustion, or prolonged repolarization in the pathologically changed nerve may be some factors of importance, perhaps by making the nerve impulse too weak to initiate a further 
propagation after a branching point or along an abnormal nerve branch. The systematic change of interspike intervals at consecutive discharges may indicate some cumulative process. Some parallels may also be drawn with the finding of Bessou, Burgess, Perl, and Taylor (1971) who described a decreasing response in C-mechanoreceptors in cats during repeated mechanical stimulation in the receptive field. The same type of findings with exhaustion at iterative mechanical and electrical stimulation have now also been made in human C-mechanoreceptors (Hallin and Torebjörk, 1970; Torebjörk and Hallin, 1970), where the degree of blocking is positively correlated to stimulation frequency. Obviously these thin and unmyelinated nerve structures behave in a way similar to some nerve twigs at reinnervation.

Are the neurogenic blockings localized only in the nerve tree where it is still branching or may they also happen in more terminal parts? These parts are the last to be myelinated at reinnervation and are also thinner and they ought therefore to offer the lowest safety factor for transmission. In the present recordings, there was often block in only one spike component, reacting to high frequency and edrophonium like the recordings with 'paired blockings'. Here the block naturally could be situated in a proximal part of the nerve twig, providing the recording were made from only one of the muscle fibres innervated by a badly conducting nerve fibre. It may, however, also be explained by a block in the very terminal nerve twigs. Possibly the greatest uncertainty during reinnervation is in the most peripheral parts, sometimes extending proximally over the last branching points of the nerve twig and so giving the neurophysiological possibility of confirming a neurogenic transmission failure in the form of 'paired blocking'. Thus, part of the jitter and blockings seen in single spikes in the compound action potentials in muscles undergoing reinnervation may be of neurogenic origin. The 'coupling discharge' in neurogenic muscular atrophy reported by Takahashi (1966) - that is, a small mono- or biphasic intermittently blocking spike in a compound action potential-could thus indicate a neurogenic block.

Might the described sensitivity to high innervation frequencies with increasing blockings be associated with the increased fatigue during activity clinically experienced in neuromuscular disorders, or is the degree of blocking usually of minor importance quantitatively? It is planned to investigate this.

Neurogenic blocking may also sometimes be responsible for a decreasing muscle response at nerve stimulation, wrongly interpreted as 'myasthenic reaction'.

It cannot at present be stated with certainty from a recording with single fibre blocking whether the block is neurogenic or situated in the neuromuscular junction or in the muscle fibre. One indication favouring neurogenic $\stackrel{9}{?}$ involvement might be the finding of a tendency to a systematic change in the interpotential $\frac{\bar{\sigma}}{\bar{N}}$ intervals instead of a quite random one as seen after curare or in myasthenia gravis. Further- $\varrho$ more, tests with, for example, edrophonium can ${ }^{\text {c }}$ be made when trying to sort out the neuro- $\vec{\circ}$ muscular block, but a positive effect can not $\overrightarrow{\vec{\omega}}$ fully exclude a neurogenic cause for the block. $\stackrel{\omega}{\circ}$ It is, therefore, now of interest to discover other tests to increase the chances of differentiating between neurogenic, neuromuscular, and myo- or genic block.

The investigation was supported by the Swedis Medical Research Council (Grant No. 14X-13\%) (Stålberg) and the Deutsche Forschungsgemeinschaft (Thiele).

\section{REFERENCES}

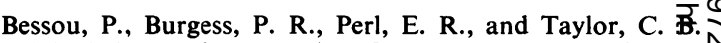
(1971). Dynamic properties of mechanoreceptors with unmyelinated (C) fibres. Journal of Neurophysiology, 34, 116131.

Blom, S., and Ringqvist, I. (1971). Neurophysiological findings in myasthenia gravis. Single muscle fibre activity in $\bar{O}$ relation to muscular fatiguability and response to anti-0 cholinesterase. Electroencephalography and Clinical Neurophysiology, 30, 477-487.

Ekstedt, J. (1964). Human single muscle fiber action poten- $\overline{\bar{O}}$ tials. Acta Physiologica Scandinavica, 61, Suppl. 226.

Ekstedt, J., and Stålberg, E. (1963). A method for recording extracellular action potentials of single muscle fibres and measuring their propagation velocity in voluntarily activated human muscle. Bulletin of the American Association of EMG Electrodiagnosis, 10, 16.

Ekstedt, J., and Stålberg, E. (1967). Myasthenia gravis. Diagnostic aspects by a new electrophysiological method. 3 Opuscula Medica, 12, 73-76.

Ekstedt, J., and Stålberg, E. (1969). Abnormal connectionsō between skeletal muscle fibers. Electroencephalography and $\bar{\beta}$ Clinical Neurophysiology, 27, 607-609.

Gilliatt, R. W. (1966). Nerve conduction in human and experimental neuropathies. Proceedings of the RoyaD Society of Medicine, 59, 989-993.

Hallin, R. G., and Torebjörk, H. E. (1970). Afferent and efferent $\mathrm{C}$ units recorded from human skin nerves in situ. Acta Societatis Medicorum Upsaliensis, 75, 277-281.

Harreveld, A. van (1952). Re-innervation of paretic muscle by collateral branching of the residual motor innervation Journal of Comparative Neurology, 97, 385-407.

Kugelberg, E., Edström, L., and Abbruzzese, M. (1970) Mapping of motor units in experimentally reinnervated rae 
muscle. Journal of Neurology, Neurosurgery, and Psychiatry, 33, 319-329.

Okamoto, M., and Riker, W., Jnr. (1969). Subacute denervation: a means of disclosing mammalian motor nerve terminals as critical sites of acetylcholine and facilitory drug actions. Journal of Pharmacology and Experimental Therapeutics, 166, 217-224.

Stålberg, E. (1966). Propagation velocity in human muscle fibers in situ. Acta Physiologica Scandinavica, 70, Suppl. 287.

Stålberg, E., Ekstedt, J., and Broman, A. (1971). The electromyographic jitter in normal human muscles. Electro- encephalography and Clinical Neurophysiology. 31, 429438.

Takahashi, K. (1966). The coupling discharge in neurogenic muscular atrophy. Archives of Neurology, 14, 617-623.

Torebjörk, H. E., and Hallin, R. G. (1970). C-fibre units recorded from human sensory nerve fascicles in situ. Acta Societatis Medicorum Upsaliensis, 75, 81-84.

Wohlfart, G. (1957). Collateral regeneration from residual motor nerve fibers in amyotrophic lateral sclerosis. Neurology (Minneap.), 7, 124-134.

Wohlfart, G. (1958). Collateral regeneration in partially denervated muscles. Neurology (Minneap.), 8, 175-180. 